\title{
Probe-based confocal laser endomicroscopy in diagnosis of desquamative interstitial pneumonia in nonsmoker
}

\author{
Igor S. Mamenko1,2, Igor V. Vasilev', Irina A. Tabanakova ${ }^{1}$, Sultan M. Gasanmagomedov ${ }^{1}$, \\ Vera V. Sysoeva ${ }^{1}$, Alexey D. Ushkov ${ }^{1}$, Tatiana A. Novickaya ${ }^{1,2}$, Piotr K. Yablonskiii, ${ }^{1,2}$ \\ ${ }^{1}$ St. Petersburg Research Institute of Phthisiopulmonology, Ministry of Health of Russia, St. Petersburg; ${ }^{2}$ Medical \\ Faculty, St. Petersburg State University, St. Petersburg, Russia
}

\begin{abstract}
Desquamative interstitial pneumonia is a rare disease that is predominantly associated with smoking. Surgical biopsy is usually recommended for the diagnosis of this disorder. Probe-based confocal laser endomicroscopy (pCLE) is a new method of minimally invasive in vivo microscopic image of airways and alveoli. We use this method for desquamative interstitial pneumonia in nonsmoker. The pCLE image shows thickened intra-alveolar septae and clusters of autofluorescent cells within the alveoli, which is unusual for non-smoking patients. We think this pattern can be
\end{abstract}

Correspondence: Igor Sergeevich Mamenko, Department of Interventional Pulmonology, St. Petersburg Research Institute of Phthisiopulmonology, Ligovskiy Ave. 2-4, Saint-Petersburg 191036, Russia.

Tel. +7.9217790109.

E-mail: dr.mamenko.is@gmail.com

Key words: confocal laser endomicroscopy; optical biopsy; interstitial lung diseases; idiopathic interstitial pneumonias; desquamative interstitial pneumonia.

Statement of Ethics: The use of pCLE was approved by the local ethical committee and the patient gave informed consent to participate.

Conflict of interest: The authors have no conflict of interest to declare.

Contributions: ISM, IAT, study concept and design; ISM, IVV, SMG, performing of the experiment; ISM, IVV, IAT, ADU, TAN, results analysis and interpretation; TAN, sample preparation; SMG, ADU, VVS, TAN, contribution to research design / implementation, results analysis, manuscript drafting; PKY, project supervision. All authors discussed the results and contributed to the final manuscript.

Received for publication: 20 February 2019.

Accepted for publication: 5 July 2019.

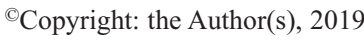

Licensee PAGEPress, Italy

Monaldi Archives for Chest Disease 2019; 89:1052

doi: 10.4081/monaldi.2019.1052

This article is distributed under the terms of the Creative Commons Attribution Noncommercial License (by-nc 4.0) which permits any noncommercial use, distribution, and reproduction in any medium, provided the original author(s) and source are credited. used for differential diagnosis of desquamative interstitial pneumonia in nonsmokers.

\section{Introduction}

Desquamative interstitial pneumonia (DIP) is a rare disease from the group of idiopathic interstitial pneumonias [1]. This disorder was first described by Liebow in 1965 and called DIP because of the belief that the main histological feature was the desquamation of epithelial cells into alveoli [2]. Nowadays it is characterized by the accumulation of numerous pigmented macrophages within most of the distal airspace of the lung and, sometimes, the presence of giant cells [3]. DIP is usually associated with tobacco smoke and is classified as smoking-related idiopathic interstitial pneumonia [1]. But it can also occur in patients exposed to certain inhaled toxins (occupational exposure) and drugs, or in certain viral illnesses and autoimmune diseases [3]. According to scientific literature, approximately 10$42 \%$ of patients with DIP are nonsmokers. [3]. Differential diagnosis of DIP is difficult and transbronchial lung biopsy has a low diagnostic yield, thus surgical biopsy is required to make a confident diagnosis [4]. However, surgical lung biopsy could have complications such as prolonged air leak, acute exacerbation or even death especially in patients with immunocompromised status, mechanical ventilation dependence, or severe respiratory dysfunction $[5,6]$. Probe-based confocal laser endomicroscopy (pCLE) is a new method of minimally invasive in vivo morphological diagnosis of airways and alveoli (so called "optical biopsy") [7]. It is useful for a diagnosis of rare lung diseases such as, for example, alveolar proteinosis and metastatic pulmonary calcification $[8,9]$.

We report a case of a diagnosis of DIP in nonsmoker using confocal laser endomicroscopy.

\section{Case Report}

A 59-year-old female was admitted to the Center of Thoracic Surgery of the "St. Petersburg Research Institute of Phthisiopulmonology" with complaints of shortness of breath with minimal exertion, moderate weakness, cough, fever by evening. From the anamnesis it is known that similar symptoms appeared for the first time in 2014, and since then she was treated with a diagnosis of pneumonia in several hospitals. Also it was known that the patient was a nonsmoker, who has been 
working for 15 years by a pig farm, and thereafter worked with cleaning products and ironing.

The patient completed a full range of examinations. Blood analysis showed mild signs of inflammation. Microbiology of sputum showed no bacteria. Spirometrical indicators were within normal limits. Computer tomography (CT) scan demonstrated multiple mosaic ground glass opacities and lymphadenopathy (Figure 1). By CT data, it was mostly resembled hypersensitivity pneumonitis, nonspecific interstitial pneumonia or alveolar proteinosis. Standard bronchoscopy revealed some signs of bronchitis and atrophy. To clarify the diagnosis, it was decided to perform a probebased confocal laser endomicroscopy by Cellvizio system (Mauna Kea Technologies, Paris, France) and transbronchial biopsy.

The pCLE was performed during the bronchoscopy under the local anesthesia of $2 \%$ lidocaine. Probe Alveoflex was inserted through the working channel of the endoscope into the distal airways of the affected segments. In all areas studied, the pCLE image showed thickened intra-alveolar septae and a large number of blending autofluorescent cells (average diameter $20 \mu \mathrm{m}$ ) in alveoli (Figure 2). The pCLE image after bronchoalveolar lavage in one of the pathological zones did not show any changes. Transbronchial biopsy was obtained from an area of the largest accumulation of autofluorescence cells by pCLE data.

Histological examination of samples of transbronchial biopsy revealed focal accumulation of macrophages and lymphocytes on the background of moderate fibrosis. Unfortunately, tissue samples were too small to determine the uniformity of lung tissue involvement in the pathological process. The obtained histological picture could also correspond to respiratory bronchiolitis-interstitial lung disease, organizing pneumonia and other interstitial pneumonias. In this regard thoracoscopic lung biopsy was obtained. Histological examination of surgical biopsy samples showed picture typical for desquamative interstitial pneumonia: multiple clusters of macrophages and alveolar cells type II in the alveolar space, uniform thickening of the interalveolar septae (Figure 3). There was no dusty pigment in macrophages, which is usual for smokers. Diagnosis of desquamative interstitial pneumonia was established by multidisciplinary consensus of specialists by the data of all examinations. After that, the patient was prescribed the correct treatment.

\section{Discussion}

Due to difficulties in diagnosis of DIP surgical lung biopsy is usually recommended [4]. The pCLE is a minimally invasive method of morphological diagnosis of lung tissue that could give some additional information about diagnosis. It is known that macrophages are visualized by pCLE mostly due to accumulation of tobacco-tar-induced fluorescence [7]. But there are some conditions when we can see highly autofluorescent cells in nonsmokers by pCLE, for example amiodarone-related pneumonia or acute lung allograft rejection [10,11]. Most authors supposed that these cells are so called activated macrophages or/and alveolar type II cells [11].

We used pCLE in nonsmoking patient with DIP and found clusters of autofluorescent cells within the alveoli in all studied areas. At the same time, the samples obtained during transbronchial biopsy also contained focal accumulation of macrophages. But specimens

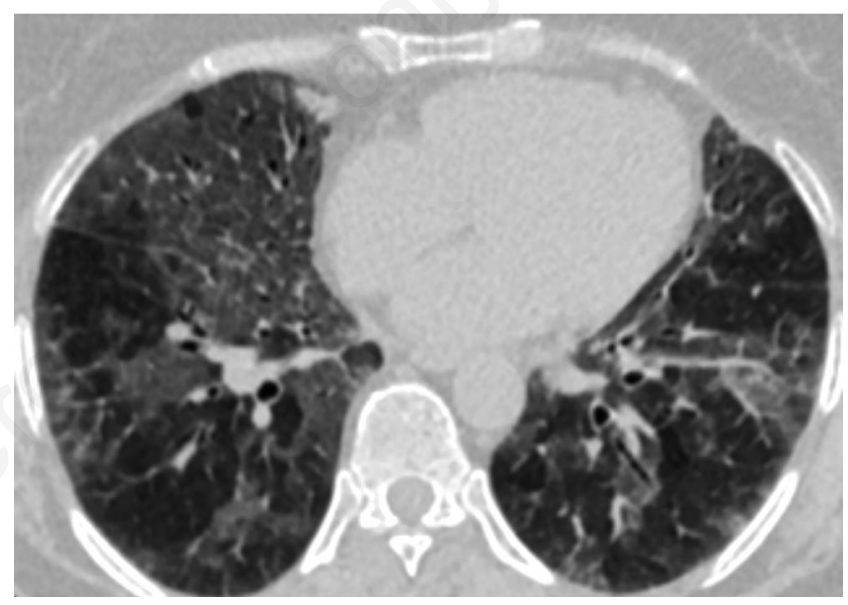

Figure 1. CT images showing patchy and mosaic areas of ground glass opacity and mild reticulation.
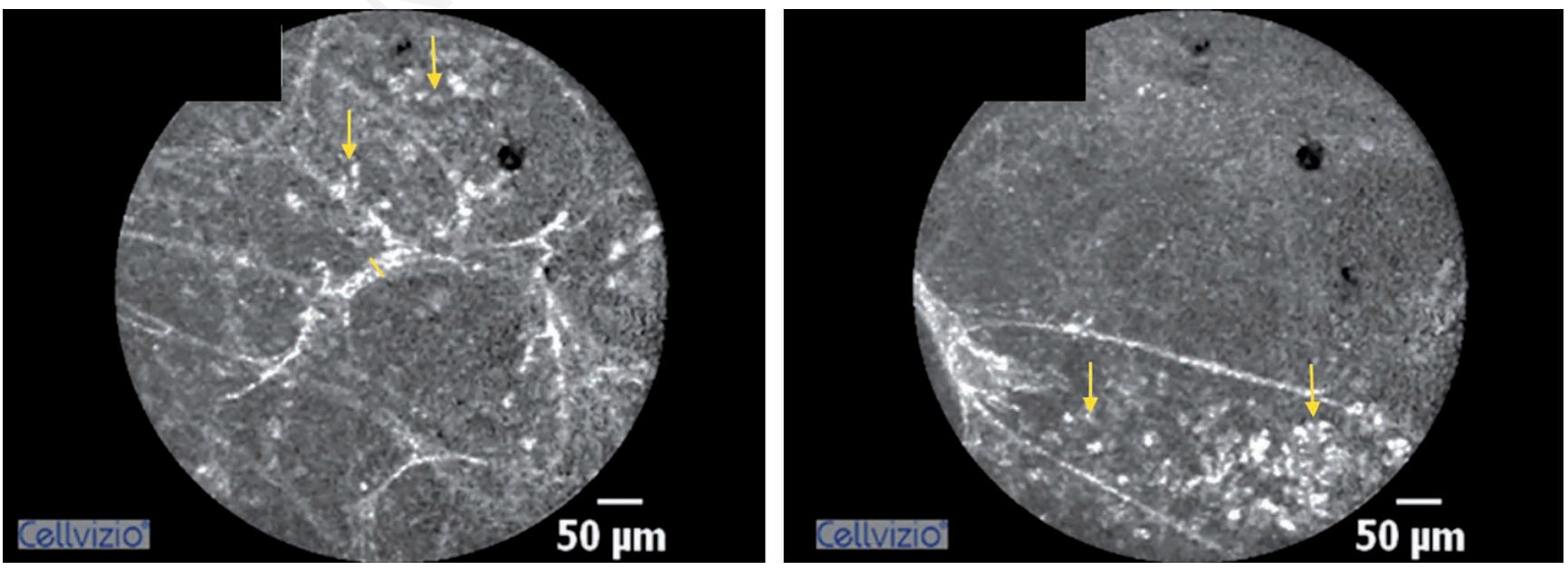

Figure 2. The pCLE image of alveoli in DIP. Alveolar structures with thickened intra-alveolar septae (yellow line) and clusters of autofluorescent cells within (yellow arrows) are visualized. 

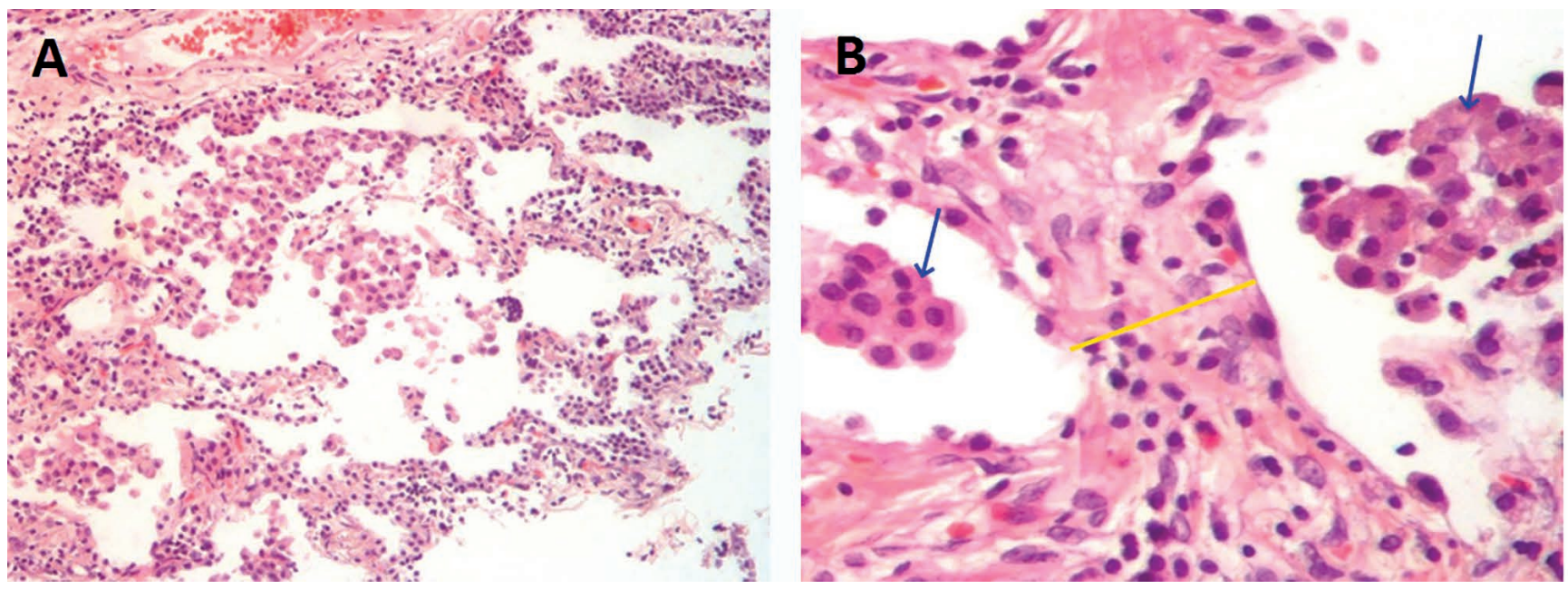

Figure 3. Histological examination of thoracoscopic biopsy sample revealed moderate fibroplastic thickening of the interalveolar septae (yellow line), large number of alveolar type II cells and macrophages (blue arrows) in alveoli (H\&E staining). Magnifications: A) 100x, B) 200x.

were too small to determine how uniform the presented changes were. A larger thoracoscopic biopsy sample proved the uniformity of pathological changes in lung tissue.

Thus, we proved the correlation between the pCLE image and the histopathological picture in DIP. We found only one research in scientific literature with pCLE image of DIP without reference about smoking status of a patient [12]. Some authors have proven that the hypercellular pattern (presence of a large number of autofluorescent cells) is less frequent in chronic fibrosing pneumonias [13]. But we found that DIP is characterized by such pattern, and think that it can be used in the differential diagnosis. Perhaps pCLE can also contribute to the correct interpretation of the histological pattern of transbronchial biopsy samples. Of course it is only one case and more research is needed to confirm this. To sum up, we think that the presence of autofluorescent cells in pCLE image in DIP in nonsmokers could give some additional information for differential diagnosis of interstitial lung diseases.

\section{References}

1. Travis WD, Costabel U, Hansell DM, et al. An official American Thoracic Society/European Respiratory Society statement: update of the international multidisciplinary classification of the idiopathic interstitial pneumonias. Am J Respir Crit Care Med 2013;188:733-48.

2. Liebow AA, Steer A, Billingsley JG. Desquamative interstitial pneumonia. Am J Med 1965;39:369-404.

3. Godbert B, Wissler MP, Vignaud JM. Desquamative interstitial pneumonia: an analytic review with an emphasis on aetiology. Eur Respir Rev 2013 1;22:117-23.

4. Bradley B, Branley HM, Egan JJ, et al. Interstitial lung disease guideline: the British Thoracic Society in collaboration with the Thoracic Society of Australia and New Zealand and the Irish Thoracic Society. Thorax 2008;63:v1-58.

5. Rotolo N, Imperatori A, Dominioni L, et al. Efficacy and safety of surgical lung biopsy for interstitial disease. Experience of 161 consecutive patients from a single institution in Italy. Sarcoidosis Vasc Diffuse Lung Di. 2015;32:251-8.

6. Han Q, Luo Q, Xie JX, et al. Diagnostic yield and postoperative mortality associated with surgical lung biopsy for evaluation of interstitial lung diseases: A systematic review and metaanalysis. J Thorac Cardiovasc Surg 2015;149:1394-401.e1

7. Thiberville L, Salaun M, Lachkar S, et al. Human in vivo fluorescence microimaging of the alveolar ducts and sacs during bronchoscopy. Eur Respir J 2009;33:974-85.

8. Danilevskaya O, Averyanov A, Lesnyak V, et al. Confocal laser endomicroscopy for diagnosis and monitoring of pulmonary alveolar proteinosis. J Bronchology Interv Pulmonol 2015;22: $33-40$.

9. Vasilev I, Mamenko I, Tabanakova I et al. Probe-based confocal laser endomicroscopy in metastatic pulmonary calcification. J Bronchology Interv Pulmonol 2018;25:60-2.

10. Salaün M, Roussel F, Bourg-Heckly G, et al. In vivo probebased confocal laser endomicroscopy in amiodarone-related pneumonia. Eur Respir J 2013;42:1646-58.

11. Yserbyt J, Dooms C, Janssens W, et al. Probe-based confocal laser endomicroscopy in acute lung allograft rejection. Thematic Poster 2442, European Respiratory Society Congress 2011, Amsterdam.

12. Wijmans L, d'Hooghe JN, Bonta PI, et al. Optical coherence tomography and confocal laser endomicroscopy in pulmonary diseases. Curr Opin Pulm Med 2017;23:275-83.

13. Meng P, Tan GL, Low SY, et al. Fibred confocal fluorescence microscopy in the diagnosis of interstitial lung diseases. J Thorac Dis 2016;8:3505-14. 
